\title{
Barriers to physical activity among African American breast cancer survivors
}

\author{
Gideon Oyekanmi ${ }^{1,2}$ and Raheem J. Paxton ${ }^{3,{ }^{*}}$ \\ ${ }^{1}$ Hightower High School, Houston, TX, USA \\ ${ }^{2}$ Dorothy I. Height Center for Health Equity \& Evaluation Research, University of Texas MD \\ Anderson Cancer Center, Houston, TX, USA \\ ${ }^{3}$ Department of Behavioral \& Community Health, School of Public Health, The University of North \\ Texas Health Science Center, Fort Worth, TX, USA
}

Dear Editor,

\section{Introduction}

African American (AA) breast cancer survivors (BCSs) have high rates of overweight/ obesity, comorbid conditions, and deficits in functional status that may contribute to poor cancer-specific and overall survival rates [1,2]. Participating in regular physical activity (PA) has tangible benefits, including weight maintenance, improvements in quality of life, functional status, and reductions in fatigue and nausea [3]. PA is also associated with a reduction in cancer recurrence and improvements in overall survival [4]. Despite the benefits associated with PA, many AA BCSs do not meet the guidelines for PA (i.e., $150 \mathrm{~min}$ of moderate to vigorous $\mathrm{PA} /$ week) [2].

African American BCSs' failure to meet PA guidelines may be due to barriers that prevent them from being active. However, limited data exist on the barriers to PA in this population. Common barriers observed in these studies included pain, fatigue, lack of social support, lack of discipline, and safety concerns [5-7]. Although these studies provided important data, the sample sizes were very small. Additional studies are needed, which examine the barriers to PA in a large sample of AA BCSs; such studies will enable subgroup comparisons and provide meaningful data on what subgroups are in the greatest need of intervention. Therefore, the purpose of this study was to characterize the PA barriers of AA BCSs and determine whether the barriers to PA differed in various subpopulations. The present study builds off a previous study that assessed the lifestyle behavior of 470 AA BCSs affiliated with the Sisters Network.

\footnotetext{
Copyright @ 2014 John Wiley \& Sons, Ltd.

*Correspondence to: Department of Behavioral \& Community Health, School of Public Health, University of North Texas Health Science Center, 3500 Camp Bowie Blvd, EAD 709L, Fort Worth TX, USA. Raheem.Paxton@UNTHSC.edu.

Conflicts of interest

The authors have conflicts of interest to disclose.
} 


\section{Methods and materials}

\section{Study population}

African American BCSs from the Sisters Network Inc., a national organization (44 chapters) of AA BCSs, participated in this needs assessment survey. The methods describing the recruitment methods were described elsewhere [2]. A total of 273 out of the 475 BCSs who initiated our Web-based survey are described here. Eligibility criteria included a previous breast cancer diagnosis, staged I to IIIC, and between 18 and 80 years old. Institutional Review Board approval was obtained prior to data collection.

\section{Measures}

Physical activity barriers-Participants were encouraged to rate barriers to PA on a five-point Likert-type response scale ranging from 1 (not at all) to 5 (very often) [8]. The 15item barrier to PA inventory consisted of items related to environmental (e.g., equipment, weather), personal (e.g., energy/fatigue), and social (e.g., company) factors. Each barrier was evaluated individually, rather than collapsed in collective sub-scales. The internal consistency reliability for the measure was $\alpha=0.92$.

Physical activity-Physical activity was assessed via a self-administered instrument designed for the Women's Health Initiative [9]. PA was calculated separately for light metabolic equivalent of task (MET level <3.0), moderate (MET level 3.0-5.9), and vigorous (MET level 86.0 ) activities. We also generated a variable to account for moderate to vigorous PA (MET level 23.0 ). This dichotomous variable ('meeting or not meeting PA guidelines') determined whether women were exercising approximately $150 \mathrm{~min}$ per week, consistent with recommended levels.

Other items-Participants' self-reported height and weight were used to compute their body mass index $\left(\mathrm{kg} / \mathrm{m}^{2}\right)$. Body mass index risk categories (normal/overweigh versus obese) were used to distinguish women. Participants also self-reported sociodemographic and medical data.

\section{Statistical analysis}

Descriptive statistics were used to summarize the medical and sociodemographic characteristics. Next, a series of paired t-tests were used to determine whether mean differences in PA barriers existed by age group, years since diagnosis, obesity status, and education level. Finally, an adjusted logistic regression model was used to evaluate the relationship between meeting current guidelines for PA and each PA barrier, adjusting for covariates (i.e., age, number of comorbidities, education, stage at cancer diagnosis, years out from diagnosis, and body mass index). Odds ratios (ORs) and 95\% confidence intervals were computed. All statistical tests were two-sided and statistical significance was determined at $p<0.05$. 


\section{Results}

\section{Sociodemographic characteristics}

The mean age of the participants was 54 years, and the mean time since diagnosis was 7 years. Many (66\%) women were diagnosed at stages II or greater. About $78 \%$ of the participants were married, and about 50\% of them had at least a college education. Many women reported hypertension (50\%), hyperlipidemia (30\%), and diabetes (30\%). Approximately, $48 \%$ of the participants were obese and less than half (44.6\%) were meeting PA guidelines. The most commonly reported barriers to PA were a lack of discipline $(43.8 \%)$, time (32.4\%), energy (34.1\%), interest (28.2\%), and good health (17.9\%).

\section{Participant characteristics and barriers}

Younger women reported significantly higher mean scores for having a lack of time and energy than did older women (all $p<0.05$, Table 1). Non-college-educated women reported significantly higher mean scores for feeling discouraged and having a fear of injury, and a lack of company, equipment, facilities or space, knowledge of how to exercise, good health, and energy than did college-educated women (all $p<0.05$ ). Obese women reported significantly higher mean scores for feeling discouraged and having a lack of facilities or space (all $p<0.05$ ). No significant mean differences were observed with regard to disease stage at diagnosis (data not shown) or time since diagnosis (all $p>0.05$ ).

\section{Meeting guidelines to physical activity and barriers}

In adjusted models, women who were not meeting guidelines for PA were more likely than women who were meeting guidelines for PA to report the following barriers: lack of interest in exercise ( $\mathrm{OR}=6.8)$, lack of self-discipline ( $\mathrm{OR}=3.9)$, lack of time $(\mathrm{OR}=3.6)$, fear of injury $(\mathrm{OR}=3.3)$, and lack of company $(\mathrm{OR}=3.1)$. The remaining associations between meeting not meeting guidelines and PA barriers are reported in Table 2.

\section{Discussion}

In this study, we found that there are certain segments of the population that are at risk for not meeting current guidelines to PA because of specific PA barriers. In particular, women who did not receive a college education appeared to be at the greatest risk and reported the most barriers. Obesity appears to be another factor that cooccurs with substantial PA barriers. Overall, obesity and less education increase one's perception of being at risk for a specific barrier.

The most prevalent barriers to PA in the study population were a lack of discipline, energy, time, and interest. The barriers observed here were similar to those observed in previous studies of AA BCSs [5-7]. The lack of interest may contribute to excessive weight gain following diagnosis and further discomfort with their weight [6]. AA BCSs may not be aware of the benefits of PA when compared with other challenges. Helping them realize that regular PA will not only improve weight but also help to manage comorbid conditions, and mood may encourage them to be more active. Promoting activities that AA BCSs prefer may 
bolster enjoyment for PA and may enhance self-efficacy to adopt and maintain regular PA over time [10].

Fear of injury and lack of good health were barriers that may refer to barriers of pain and fatigue reported from previous studies [5-7]. Such concerns may co-occur with number of comorbidities, a correlate of PA in this population [2]. Interventions targeting women with injuries, a fear of injuries, or comorbidities could focus on interrupting prolonged periods of sedentary time, chair-based exercises, yoga, or stretching at home. Such strategies may build self-efficacy and gradually promote behavior change over time.

Less-educated women more prone to barriers. These data correspond to our previous study suggesting that less education was indicative of not meeting PA guidelines [2]. Lower socioeconomic is a known correlate of PA; however, PA can be acquired at little to no cost in any environment. Studies educating AA BCSs of convenient and inexpensive PA strategies are needed.

This study had several notable strengths. It is modest in size, provides data on an underrepresented population, and identifies subgroups who may be more in need of PA interventions. However, the findings of this study should be considered with awareness of its limitations. The data were self-reported with potential recall and reporting biases. In addition, the participant response rate was relatively low compared with the available pool of AA BCSs $(\mathrm{n} \sim 3800)$ in the Sisters Network database.

In conclusion, this study identified subgroups of AA BCSs that may be in the greatest need for intervention. These findings are similar to those observed in healthy population and will be useful for researchers and health professionals in attempting to develop lifestyle interventions in this underserved population. Given that barriers of discipline, time, and social support contribute to lower levels of PA in this population, group or family-based interventions may be warranted. Additionally, gradually reducing and interrupting prolonged periods of sedentary behavior and assisting women in the transition to a more active lifestyle may help to curb the significant comorbid conditions experienced by these women.

\section{Acknowledgments}

We thank the women of Sisters Network, Inc., for participating in our study and Scientific Publication Department at MD Anderson for editing our manuscript. We would also like to thank Ms. Kelly Hodges of the Sisters Network for playing an instrumental role in organizing the recruitment efforts. This research was supported in part by National Cancer Institute grants K01CA158000 (to R. J. P.) and by the National Institutes of Health through MD Anderson Cancer Center's Support Grant (CA016672).

Financial disclosures

The authors have no financial disclosures.

\section{References}

1. Paxton RJ, et al. Associations among physical activity, body mass index, and health-related quality of life by race/ethnicity in a diverse sample of breast cancer survivors. Cancer. 2012; 118(16):40244031. [PubMed: 22252966]

2. Paxton RJ, et al. Lifestyle behaviors of African American breast cancer survivors: a Sisters Network, Inc. study. PLoS One. 2013; 8(4):e61854. [PubMed: 23626740] 
3. Speck RM, et al. An update of controlled physical activity trials in cancer survivors: a systematic review and meta-analysis. J Cancer Surviv. 2010; 4(2):87-100. [PubMed: 20052559]

4. Ibrahim EM, Al-Homaidh A. Physical activity and survival after breast cancer diagnosis: metaanalysis of published studies. Med Oncol. 2011; 28(3):753-765. [PubMed: 20411366]

5. Stolley MR, et al. Health behaviors and breast cancer: experiences of urban African American women. Health Educ Behav. 2006; 33(5):604-624. [PubMed: 16923833]

6. Weathers B, et al. Perceptions of changes in weight among African American breast cancer survivors. Psychooncology. 2006; 15(2):174-179. [PubMed: 15937868]

7. Spector D, Battaglini C, Groff D. Perceived exercise barriers and facilitators among ethnically diverse breast cancer survivors. Oncol Nurs Forum. 2013; 40(5):472-480. [PubMed: 23989021]

8. Hovell MF, et al. Identifying correlates of walking for exercise: an epidemiologic prerequisite for physical activity promotion. Prev Med. 1989; 18(6):856-866. [PubMed: 2626418]

9. Langer RD, et al. The Women's Health Initiative Observational Study: baseline characteristics of participants and reliability of baseline measures. Ann Epidemiol. 2003; 13(9 Suppl):S107-S121. [PubMed: 14575943]

10. Rogers LQ, et al. Correlates of physical activity self-efficacy among breast cancer survivors. Am J Health Behav. 2008; 32(6):594-603. [PubMed: 18442339] 


\section{Key points}

- Few AA BCSs meet current guidelines to physical activity, yet little is known about the barriers that prevent them from being active.

- AA BCSs frequently reported barriers of lack of discipline, time, energy, interest, and good health.

- PA barriers among AA BCSs did not differ according to time out from diagnosis or stage of diagnosis.

- AA BCSs who were obese and less educated experienced the greatest number of physical activity barriers.

- Lack of interest in physical activity, discipline, and support significantly increased one's risk for not meeting current guidelines to physical activity. 
Table 1

Barriers to physical activity by sociodemographic and lifestyle characteristics

\begin{tabular}{|c|c|c|c|c|c|c|c|c|c|}
\hline \multirow[b]{2}{*}{ Reported barriers } & \multicolumn{3}{|c|}{ Age group $N=273$} & \multicolumn{3}{|c|}{ Body size $N=273$} & \multicolumn{3}{|c|}{ Education $N=270$} \\
\hline & $\begin{array}{c}<55 \\
\text { years } \\
\text { M }(S D)\end{array}$ & $\begin{array}{c}>55 \\
\text { years } \\
\text { M (SD) }\end{array}$ & $p$-value & Non-obese M (SD) & Obese M (SD) & $p$-value & $\begin{array}{c}\text { Non- } \\
\text { college } \\
\text { graduates } \\
\mathbf{M}(\mathrm{SD})\end{array}$ & $\begin{array}{c}\text { College } \\
\text { graduates } \\
\text { M (SD) }\end{array}$ & $p$-value \\
\hline $\begin{array}{l}\text { Lack of interest in } \\
\text { exercising }\end{array}$ & $2.7(1.2)$ & $2.9(1.3)$ & 0.21 & $2.7(1.3)$ & $2.9(1.2)$ & 0.28 & $2.9(1.3)$ & $2.7(1.3)$ & 0.18 \\
\hline Lack of self-discipline & $3.1(1.3)$ & $3.2(1.3)$ & 0.93 & $3.0(1.3)$ & $3.3(1.2)$ & 0.12 & $3.2(1.3)$ & $3.1(1.2)$ & 0.41 \\
\hline Lack of time & $3.1(1.2)$ & $2.7(1.2)$ & 0.01 & $2.9(1.3)$ & $3.0(1.1)$ & 0.60 & $2.9(1.2)$ & $3.0(1.2)$ & 0.44 \\
\hline Lack of energy & $3.3(1.2)$ & $2.8(1.1)$ & 0.00 & $3.0(1.2)$ & $3.2(1.2)$ & 0.13 & $3.2(1.2)$ & $3.0(1.2)$ & 0.23 \\
\hline Lack of company & $2.3(1.3)$ & $2.2(1.2)$ & 0.58 & $2.2(1.3)$ & $2.3(1.2)$ & 0.46 & $2.5(1.3)$ & $2.0(1.2)$ & 0.00 \\
\hline Lack of enjoyment & $2.3(1.2)$ & $2.5(1.1)$ & 0.13 & $2.3(1.2)$ & $2.5(1.1)$ & 0.21 & $2.5(1.2)$ & $2.3(1.1)$ & 0.08 \\
\hline Discouragement & $2.2(1.3)$ & $2.3(1.2)$ & 0.86 & $2.0(1.1)$ & $2.5(1.3)$ & 0.00 & $2.4(1.3)$ & $2.1(1.1)$ & 0.04 \\
\hline Lack of equipment & $2.1(1.3)$ & $2.1(1.2)$ & 0.73 & $2.0(1.2)$ & $2.2(1.3)$ & 0.19 & $2.3(1.3)$ & $1.9(1.1)$ & 0.00 \\
\hline Lack of good weather & $2.2(1.0)$ & $2.3(1.1)$ & 0.57 & $2.2(1.0)$ & $2.3(1.1)$ & 0.51 & $2.4(1.1)$ & $2.1(1.0)$ & 0.08 \\
\hline Lack of skills & $2.0(1.1)$ & $2.0(1.0)$ & 0.77 & $1.9(1.0)$ & $2.1(1.1)$ & 0.16 & $2.1(1.2)$ & $1.8(1.0)$ & 0.02 \\
\hline $\begin{array}{l}\text { Lack of facilities or } \\
\text { space }\end{array}$ & $2.0(1.2)$ & $2.0(1.2)$ & 0.94 & $1.9(1.1)$ & $2.2(1.3)$ & 0.04 & $2.2(1.4)$ & $1.8(1.0)$ & 0.01 \\
\hline $\begin{array}{l}\text { Lack of knowledge of } \\
\text { how to exercise }\end{array}$ & $2.1(1.2)$ & $2.0(1.1)$ & 0.66 & $2.0(1.1)$ & $2.1(1.3)$ & 0.24 & $2.2(1.3)$ & $2.0(1.0)$ & 0.03 \\
\hline Lack of good health & $2.5(1.3)$ & $2.3(1.2)$ & 0.18 & $2.3(1.2)$ & $2.5(1.3)$ & 0.18 & $2.5(1.3)$ & $2.2(1.2)$ & 0.04 \\
\hline Fear of injury & $1.9(1.2)$ & $1.8(1.0)$ & 0.45 & $1.7(1.0)$ & $2.0(1.2)$ & 0.06 & $2.0(1.3)$ & $1.7(1.0)$ & 0.03 \\
\hline
\end{tabular}

Higher mean scores indicate that the subgroups in question had a higher risk for encountering certain barriers. The response options ranged from 1 (not at all) to 5 (very often). 


\section{Table 2}

Odds associated with not meeting current guidelines to physical activity and perceiving barriers to physical activity

\begin{tabular}{lccl}
\hline & \% experiencing barrier often or very often & Odds ratio & 95\% confidence interval \\
\hline Lack of interest in exercising & 60 & 6.8 & $3.8,12.2$ \\
Lack of self-discipline & 72 & 3.9 & $2.2,7.1$ \\
Lack of time & 64 & 2.1 & $1.2,3.7$ \\
Lack of energy & 71 & 2.5 & $1.4,4.6$ \\
Lack of company & 37 & 3.6 & $2.0,6.5$ \\
Lack of enjoyment & 47 & 3.1 & $1.8,5.4$ \\
Discouragement & 37 & 2.4 & $1.4,4.3$ \\
Lack of equipment & 30 & 2.8 & $1.5,5.1$ \\
Lack of good weather & 41 & 1.7 & $1.0,3.0$ \\
Lack of skills & 30 & 1.5 & $0.8,2.6$ \\
Lack of facilities or space & 30 & 2.2 & $1.2,4.1$ \\
Lack of knowledge of how to exercise & 33 & 2.6 & $1.4,4.6$ \\
Lack of good health & 43 & 2.8 & $1.6,4.7$ \\
Fear of injury & 23 & 3.3 & $1.6,6.7$ \\
\hline
\end{tabular}

Analyses were adjusted for age, number of comorbidities, education, stage at cancer diagnosis, years out from diagnosis, and body mass index. 